\title{
La colonie française de Saint-Domingue ; Les Vengeurs du Nouveau Monde. Histoire de la révolution haïtienne
}

\section{Serge Bianchi}

\section{(2) OpenEdition \\ 1 Journals \\ Édition électronique \\ URL : https://journals.openedition.org/ahrf/7283 \\ DOI : 10.4000/ahrf.7283 \\ ISSN : 1952-403X \\ Éditeur : \\ Armand Colin, Société des études robespierristes}

\section{Édition imprimée}

Date de publication : 1 septembre 2006

Pagination : 191-195

ISSN : 0003-4436

\section{Référence électronique}

Serge Bianchi, «La colonie française de Saint-Domingue ; Les Vengeurs du Nouveau Monde. Histoire de la révolution haïtienne », Annales historiques de la Révolution française [En ligne], 345 I juilletseptembre 2006, mis en ligne le 08 juillet 2008, consulté le 01 juillet 2021. URL : http:// journals.openedition.org/ahrf/7283; DOI : https://doi.org/10.4000/ahrf.7283

Ce document a été généré automatiquement le 1 juillet 2021.

Tous droits réservés 


\title{
La colonie française de Saint- Domingue ; Les Vengeurs du Nouveau Monde. Histoire de la révolution haïtienne
}

\author{
Serge Bianchi
}

\section{RÉFÉRENCE}

François Blancpain, La colonie française de Saint-Domingue, Paris, Karthala, 2004, 244 p., ISBN 2-84586-590-2, $22 €$; Laurent Dubois, Les Vengeurs du Nouveau Monde. Histoire de la révolution haïtienne, Rennes, Les Perséides, 2005, 437 p., ISBN 2-915596-12-3, $25 €$.

1 L'histoire de la Révolution à Saint-Domingue, qui est celle de la première abolition de l'esclavage et de la première indépendance d'une colonie noire, acquiert progressivement ses lettres de noblesse. Deux ouvrages complémentaires éclairent des aspects moins connus d'une question devenue «à la mode», des années 1980 au bicentenaire de l'indépendance d'Haïti en 2004. François Blancpain, spécialisé dans les questions économiques et sociales, propose un ouvrage stimulant et ramassé sur la "révolution vue du côté haïtien", soit onze chapitres de l'Ancien Régime à l'indépendance, une bibliographie francophone d'une quarantaine d'ouvrages et une chronologie. Laurent Dubois, enseignant à l'Université du Michigan (États-Unis) tente une somme érudite sur la révolution haïtienne, en treize chapitres (plus un prologue, un épilogue, un index), agrémentés par une bibliographie considérable, tant de «sources primaires» (cinquante imprimés de la période) que « secondaires » - plus de cent vingt références, tant en langue française qu'en anglais -, soit une documentation quasi exhaustive, exemplaire pour les notes de bas de page. On doit souligner la qualité, la fluidité (et la rapidité) de la traduction (de Thomas Van Ruymbeke) par les éditions Les Perséides, au-delà de quelques pêchés de jeunesse - sur des dates de la Révolution 
française ou la traduction de deux ou trois termes comme "gens de couleur» (p. 28) alors que « libres de couleur » ou " mulâtres » conviendraient mieux.

Les deux livres abordant la même période et le même thème général, il est tentant de faire des comparaisons, afin d'estimer leurs mérites spécifiques. Ce ne sera pas l'objet de cette présentation, qui insistera plutôt sur leur complémentarité et leurs apports communs. La démarche de François Blancpain est de l'ordre de la vulgarisation pour un large public, d'une histoire "agrémentée par des formules». Parmi les formules de l'auteur : «Toute l'histoire de la République devenue indépendante sera dominée par la lutte entre les bourgeois, héritiers des colons propriétaires des grandes plantations sucrières, et les gens du peuple, descendants des esclaves, fermement partisans de la petite propriété familiale » (p. 101). De nombreuses citations font également mouche, telle l'analyse de l'interprétation du Code noir par le ministre de la Marine en 1767 : «Le don de la liberté » ne peut effacer « la première tâche [de l'esclavage] qui s'étend sur tous leurs descendants ». Si l'exposé général des faits est classique, synthétique et suggestif, la qualité principale du livre réside dans la multiplication des documents d'époque (édits, lettres, proclamations, décrets) qui confère aux analyses une touche d'authenticité. Laurent Dubois, au contraire, cite peu de documents directs, mais en conduit des analyses sophistiquées, comme celle de l'écrivain James Stephen (et non Stephens) à propos de l'expédition Leclerc de 1802. Le recours de l'un (citations) à l'autre (analyses) pour éclairer des contenus concrets est ainsi particulièrement efficace.

On trouve chez François Blancpain des mises au point efficaces sur des questions essentielles: l'historique de la traite, la montée du préjugé de couleur au rythme des affranchissements, l'ascension de Toussaint Louverture. Pourtant, le mérite particulier de l'ouvrage est d'étudier les régimes de la terre, les évolutions de la mise en valeur des plantations, de l'esclavage au salariat par les différents détenteurs du pouvoir à SaintDomingue, des planteurs blancs aux décrets de Toussaint sous le Directoire, en passant par les mesures décalées des commissaires de la République à l'été 1793, quand "l'égalitarisme jacobin» de Polverel dans le Sud s'oppose au maintien d'une exploitation sévère par Sonthonax dans le Nord, dans le cadre de la libération juridique des anciens esclaves. À quelques formules très discutables près (principalement sur Robespierre "grand prêtre du culte de l'Être Suprême "; des procès «staliniens ", p. 221), la lecture de François Blancpain est utile et constitue une bonne initiation, si elle ne bouleverse pas la trame aujourd'hui reconnue de la révolution haïtienne. On ressent à sa lecture la complexité des solutions sociales dans Saint-Domingue en révolution, le maintien de l'exploitation des anciens esclaves par les élites successives blanches et mulâtres, et l'ampleur des tragédies ouvertes par le débarquement de 60000 hommes en 1802, pour rétablir l'autorité de l'ancienne métropole et (probablement) l'esclavage.

Le livre de Laurent Dubois est différent par la démarche, la composition, les objectifs. Il s'agit d'une véritable somme relative à dix années de révolution dans l'espace caribéen, à partir d'une érudition remarquable par son ampleur et sa pertinence. L'auteur livre souvent des anecdotes significatives (telle lettre d'un régisseur, tel procès, telle révolte) à l'appui d'analyses fouillées et approfondies des thèmes brûlants. Les «vengeurs du nouveau monde », ce titre ambigu, renvoie à l'inscription de l'indépendance d'Haïti, proclamée en 1804, dans le contexte large de la colonisation outre-Atlantique par les Européens, de Colomb à Toussaint Louverture et au général Dessalines, auteur en 1804 de la formule clé : "J'ai vengé l'Amérique »(p. 403). La lecture de plusieurs chapitres 
(La fermentation; L'héritage) emporte l'adhésion par la précision des données, les nuances apportées, le souci d'une approche concrète - par exemple l'évocation de la vie des esclaves dans les habitations -. L'association d'auteurs récents (français, anglais, américains) enrichit l'interprétation de façon souvent décisive, par exemple au sujet de la mentalité des ethnies africaines, ou des circonstances de la révolte de Bois Caïman. On pourrait parler d'exhaustivité, voire d'œuménisme, tant l'ouvrage rend compte des différentes écoles et des courants historiographiques qui se sont succédés (ou affrontés) depuis un demi siècle! Quelques approximations sur l'esprit républicain, ou la date de l'abolition de l'esclavage à la Convention, quelques lacunes sur le destin de Toussaint ou le contexte métropolitain de la première abolition, ne pénalisent pas un maître livre, lorsqu'il éclaire par exemple les motivations des multiples acteurs de la guerre (civile et extérieure) en 1793-1794: planteurs blancs (Galliffet), commissaires (Sonthonax), Anglais, Espagnols, mulâtres (Rigaud), anciens esclaves (Christophe)... Laurent Dubois rejoint ou complète François Blancpain sur de nombreux points, que l'on pourra, après d'autres lectures, considérer désormais comme acquis.

5 Le plus important des apports communs a trait au régime économique des plantations, au cours de la décennie révolutionnaire. Si les destructions causées par les guerres ont ruiné l'économie domingoise et le commerce généré par la traite et l'esclavage, des permanences s'imposent dans les relations entre les propriétaires et les travailleurs des terres. Les commissaires de la République et les généraux noirs (essentiellement Toussaint) poursuivent les mêmes objectifs : le travail est nécessaire et moral; les plantations doivent tourner; les ouvriers agricoles ne doivent pas les quitter; les fruits reviennent pour l'essentiel au propriétaire, dans une proportion des deux tiers des récoltes; le régime du travail doit être assoupli à cinq ou six jours par semaine. Dans ces conditions, on ne peut parler de "révolution sociale », de début de réforme agraire, de redistribution égalitaire du sol correspondant à la liberté juridique obtenue par les quelque 500000 esclaves. De nouveaux planteurs (les élites des mulâtres, les généraux, les dynasties africaines princières) prennent la place des grands planteurs blancs (ou la partagent). Les conflits entre l'élite propriétaire noire et les ouvriers agricoles perdureront pendant le XIX ${ }^{\mathrm{e}}$ siècle, dans l'île indépendante.

6 Une autre rencontre des deux auteurs peut se faire autour des relations entre Toussaint Louverture et Sonthonax, comme baromètre des relations entre la Première République et le personnage le plus charismatique de la révolution haïtienne. Sonthonax n'attend pas l'avis de la Convention pour abolir l'esclavage dans la partie Nord de la colonie. Il le fait par stratégie, pour éviter l'encerclement (par les Anglais, les Espagnols, les esclaves, l'assemblée coloniale) et la perte l'île. Il le fait aussi par principe, en dissociant les aspects juridiques et économiques. Malgré un retour de deux ans en métropole et un rappel définitif provoqué par Toussaint sous le Directoire, il a marqué les consciences et bénéficié d'une popularité considérable auprès des esclaves affranchis. Son opinion sur Toussaint varie au fil de la prise de pouvoir par ce dernier, de l'éloge du «Spartacus noir» (propos de Laveaux) au rejet de "l'un des chefs de la Vendée de SaintDomingue » (Blancpain, p. 144), allusion à la politique catholique du général noir. Le jeu personnel de Toussaint est exposé dans les deux ouvrages: ses relations unilatérales avec l'Angleterre, l'Espagne et les États-Unis; les retour forcés et provoqués des représentants français en métropole (Laveaux, Sonthonax et Hédouville); la politique de conciliation avec les planteurs blancs, au risque d'être qualifié de " contrerévolutionnaire ", avant les accusations de dictature et de trahison à l'égard de la République au moment de l'élimination de l'armée des mulâtres de Rigaud, en 1800. 
Une démythification de la politique de Toussaint, jointe à la mise en évidence de mérites incontestables, est ainsi en marche, grâce aux apports croisés des deux ouvrages, après ceux de Pierre Pluchon.

7 Enfin, les deux livres insistent sur l'intensité et la complexité des rapports entre la métropole et l'île caraïbe. D'une part, en soulignant - moins fortement chez Laurent Dubois -, la relation dialectique entre la république jacobine-sans-culotte et la première abolition de l'esclavage, au nom des principes et d'intérêts mutuels qui font des anciens esclaves des citoyens républicains, élisant des représentants, comme Belley ou Dufay à la Convention. Ils sont moins diserts sur les échos de la marche à l'indépendance dans l'espace caraïbe et dans les autres plantations atlantiques. D'autre part, ils marquent avec force le caractère tragique (et la violence inouïe) des affrontements consécutifs à l'expédition Leclerc, qualifiée alors de " croisade contre la barbarie noire ", dans l'« intérêt de la civilisation » (Talleyrand). La pacification semble un succès en mai 1802, quand les chefs historiques (Dessalines, Henri Christophe, et le " premier des noirs ", Toussaint) acceptent un moment de négocier et de liquider les foyers insurrectionnels des combattants noirs irréductibles. Mais l'évidence de la volonté de rétablissement de l'esclavage par Bonaparte, la duplicité de Leclerc (dans l'arrestation de Toussaint) provoquent la fracture définitive : « Il ne faut plus de blancs parmi nous» (Dessalines). Les massacres sommaires, l'usage de chiens-déchiqueteurs de noirs, les guerres meurtrières des chefs noirs (Christophe contre Paul Louverture), les ravages de la fièvre jaune, la défection de certaines troupes (les Polonais!) constituent certaines des pages les plus sanglantes de l'histoire de l'époque, après les guerres de Vendée : Saint-Domingue déplore plus de 100000 morts en 1802-1803, et une population en régression au moment de l'indépendance. Le blanc est arraché du drapeau « haïtien » (nom donné au pays indépendant). La déclaration d'indépendance du 1er janvier 1804, sur le modèle américain de 1776, proclame une " haine éternelle à la France »: "Qu'avons-nous de commun avec ce peuple bourreau ». Au-delà des tentatives d'explication de cette folie ("Mon âme est flétrie » dit Leclerc avant sa mort), les ouvrages de Blancpain et de Dubois montrent que d'autres voies étaient possibles, dans l'établissement de relations commerciales et diplomatiques avec cet État noir émergent dont l'autonomie ne pouvait plus être remise en cause. C'est donc dans une perspective historique large, si on néglige les tragédies postérieures à 1804, qu'il faut interpréter la déclaration de Dessalines au moment de l'indépendance d'Haïti : «J'ai sauvé mon pays. J'ai vengé l'Amérique ».

8 Ainsi progresse l'histoire de «la colonie française de Saint-Domingue » et de la « révolution haïtienne ». Les regards complémentaires et croisés des deux historiens nous éloignent des polémiques récentes et trop médiatisées sur les antécédents et les héritages de la traite des esclaves, à une échelle mondiale. Ils rétablissent des vérités premières sur la "révolution haïtienne ", quatrième révolution aboutie de «l'ère des révolutions ", après les révolutions américaine, française, batave. La première abolition de l'esclavage est bien l'un des événements majeurs de la décennie révolutionnaire, fruit d'un ensemble dense et complexe de liens entre la métropole républicaine et une colonie déchirée par les guerres. On ne saurait négliger les luttes des abolitionnistes français et domingois, pas plus que les résistances de la majorité des planteurs blancs autour du maintien du préjugé de couleur et de «l'éternité » de l'esclavage. La guerre des esclaves a été une guerre coloniale d'un type nouveau, où s'imposent finalement la stratégie et les compétences militaires des armées noires. Le rétablissement programmé de l'esclavage a entraîné une rupture fatale et totale entre une métropole 
et sa colonie. Cette fracture, si longtemps dissimulée, ne saurait effacer l'influence de la première abolition et le chemin parcouru par les armées républicaines - noires et blanches - enfin « unies par les idéaux de liberté et d'égalité civique », entre 1793 et 1798. C'est dire l'utilité et l'efficacité pédagogique des ouvrages de François Blancpain et de Laurent Dubois, associés pour rétablir certaines vérités longtemps occultées d'un passé «qui fait mal» et qui continue de diviser des communautés et des mémoires conflictuelles. 